\title{
Electronic cigarette exposure triggers neutrophil inflammatory responses
}

\author{
Andrew Higham ${ }^{*}$, Nicholas J. W. Rattray ${ }^{2}$, Jennifer A. Dewhurst ${ }^{1}$, Drupad K. Trivedi², Stephen J. Fowler', \\ Royston Goodacre ${ }^{2}$ and Dave Singh ${ }^{1}$
}

\begin{abstract}
Background: The use of electronic cigarettes (e-cigs) is increasing and there is widespread perception that e-cigs are safe. E-cigs contain harmful chemicals; more research is needed to evaluate the safety of e-cig use. Our aim was to investigate the effects of e-cigs on the inflammatory response of human neutrophils.

Methods: Neutrophils were exposed to e-cig vapour extract (ECVE) and the expression of CD11b and CD66b was measured by flow cytometry and MMP-9 and CXCL8 by ELISA. We also measured the activity of neutrophil elastase (NE) and MMP-9, along with the activation of inflammatory signalling pathways. Finally we analysed the biochemical composition of ECVE by ultra-high performance liquid chromatography mass spectrometry.

Results: ECVE caused an increase in the expression of CD11b and CD66b, and increased the release of MMP-9 and CXCL8. Furthermore, there was an increase in NE and MMP-9 activity and an increase in p38 MAPK activation. We also identified several harmful chemicals in ECVE, including known carcinogens.

Conclusions: ECVE causes a pro-inflammatory response from human neutrophils. This raises concerns over the safety of e-cig use.
\end{abstract}

Keywords: Electronic cigarettes, COPD, Inflammation, Smoking, Neutrophils, MMP-9

\section{Background}

There are an estimated 13 million users of electronic cigarettes (e-cigs) worldwide [1]. E-cigs are used to help reduce or stop tobacco smoking [2]. However, it has been shown that toxic chemicals are present in e-cig vapour, such as formaldehyde and acrolein [3-5], casting doubt on the safety of using e-cigs.

Cigarette smoke extract increases the secretion of proinflammatory mediators from a range of different cell types including epithelial cells, macrophages and neutrophils [6-8]. Similarly, e-cig vapour exposure increases the release of inflammatory mediators from keratinocyte and alveolar epithelial cell lines [9]. Furthermore, e-liquid increases interleukin-6 secretion from bronchial epithelial cells [10]. This raises concerns over the potential of e-cigs

\footnotetext{
* Correspondence: Andrew.Higham@manchester.ac.uk

Andrew Higham and Nicholas I W Rattray are joint first authors.

'Manchester Academic Health and Science Centre, University Hospital of

South Manchester Foundation Trust, Centre for Respiratory and Allergy

Medicine, Institute of Inflammation and Repair, Faculty of Medical and

Human Sciences, The University of Manchester, Manchester, UK

Full list of author information is available at the end of the article
}

to promote pulmonary inflammation in a similar manner to tobacco smoking.

Neutrophil numbers are increased in the lungs of chronic obstructive pulmonary disease (COPD) patients and increased numbers positively correlate with disease severity $[11,12]$. Chemokine $\mathrm{C}-\mathrm{X}-\mathrm{C}$ motif ligand 8 (CXCL8) is a key neutrophil chemoattractant [13] and the levels of CXCL8 are increased in the lungs of COPD patients [14]. Cigarette smoke exposed neutrophils secrete CXCL8, which may lead to increased neutrophil recruitment to the lungs.

Neutrophils are involved in many aspects of COPD pathophysiology. For example, neutrophils release proteases such as neutrophil elastase (NE) and matrix metalloproteinase-9 (MMP-9) which cause tissue destruction resulting in emphysema [15]. Cigarette smoke stimulates NE and MMP-9 release from neutrophils $[8,16]$. The levels of NE and MMP-9 are increased in the airways of COPD patients and the levels positively correlate with disease severity [17-19]. Moreover, COPD neutrophils demonstrate a higher level of activation compared to controls \{Wright, 2016\#132\}. 
Upon exposure to cigarette smoke and the bacterial peptide $\mathrm{N}$-formylmethionyl-leucyl-phenalanine (fMLP), neutrophils increase the expression of CD11b and CD66b, important for the migration and degranulation of neutrophils at sites of inflammation [20, 21]. There is increased expression of the adhesion molecule CD11b on the surface of blood and lung neutrophils of COPD patients [22-25]. CD11b and CD66b can be regarded as markers of neutrophil activation.

The effects of e-cigs on neutrophil activity are unknown. Due to the complex mixture of chemicals present in e-cigs, we hypothesised that exposure to e-cigs will induce neutrophil activation. The aim of this study was to investigate the effects of e-cig exposure on the inflammatory response of human neutrophils. We examined the effects on CD11b, CD66b and neutrophil shape change, and the release of MMP-9, NE and CXCL8, which are all implicated in the pathogenesis of COPD $[17,26,27]$.

\section{Methods}

\section{Study subjects}

Ten healthy never-smokers were recruited to donate peripheral blood samples (demography shown in Additional file 1). All subjects gave written informed consent. This research was approved by Preston research ethics committee REC reference 10/H1016/25.

\section{Electronic cigarette}

Table 1 shows the three brands of e-cigs used for this study; $\mathrm{VIP}^{\oplus}$ (brand 1), $\mathrm{KIK}^{\oplus}$ (brand 2) and Puritane (brand 3). All experiments were performed using brand 1 containing $24 \mathrm{mg}$ nicotine unless otherwise stated.

\section{E-cig vapour extract preparation}

For brand 1 and brand 2, e-cig vapour extract (ECVE) was prepared by bubbling e-cig vapour through RPMI-1640 culture medium (Sigma-Aldrich, Poole, UK) supplemented with $2 \mathrm{mM}$ L-glutamine (Invitrogen, Paisley, UK), $100 \mathrm{U} / \mathrm{mL}$ penicillin and $100 \mu \mathrm{g} / \mathrm{mL}$ streptomycin (Sigma-Aldrich) using a Watson-Marlow 520R peristaltic pump (WatsonMarlow Ltd, Falmouth, UK). Vapour was generated by manual activation of the heating coil at a flow rate of $15 \mathrm{~mL} / \mathrm{min}$. For brand 3, vapour was generated automatically using a flow rate of $90 \mathrm{~mL} / \mathrm{min}$. Each cycle of vapour lasted $10 \mathrm{~s}$ followed by a $30 \mathrm{~s}$ interval. In total for each brand, e-cig vapour was bubbled through the media 20 times and the total time of preparation was approximately 13 mins.

For all experiments, ECVE optical density (OD) was measured using a spectrophotometer (Eppendorf, Stevenage, UK)) at the $320 \mathrm{~nm}$ wavelength, as is the case for previous publications using cigarette smoke extract [7, 28, 29]. Optical density is an arbitrary unit used to find the concentration of ECVE which gives the optimal response and to normalise preparations for the purposes of obtaining reproducible data. The choice of $320 \mathrm{~nm}$ wavelength was based upon the relative contribution of this peak in all of the ECVE preparations (Additional file 2) determined using a POLARstar Omega microplate reader (BMG Labtech, Buckinghamshire, UK); this was a major peak in the absorbance spectrum for all ECVE preparations. It was important to avoid peaks at $260 \mathrm{~nm}$ for two reasons; 1) the absorbance spectra of nicotine demonstrate a major peak at $260 \mathrm{~nm}$ [30, 31]; 2) The quantification of nucleic acids utilises absorbance at $260 \mathrm{~nm}$. Any contaminating nucleic acids may therefore affect the results at this part of the spectrum. Foetal calf serum (FCS; $10 \%$, Invitrogen) was then added and the ECVE was filtered using a syringe driven $0.22 \mu \mathrm{m}$ filter. The ECVE was then adjusted to the desired OD using culture medium.

\section{Cigarette smoke extract preparation}

Cigarette smoke extract (CSE) was prepared as previously described [7]. Briefly, one 3R4F Kentucky research cigarette (University of Kentucky, Kentucky, USA) was bubbled through RPMI-1640 culture medium supplemented with $2 \mathrm{mM}$ L-glutamine, $100 \mathrm{U} / \mathrm{mL}$ penicillin and $100 \mu \mathrm{g} / \mathrm{mL}$ streptomycin using a Watson-Marlow 520R peristaltic pump. $10 \%$ FCS was then added and the CSE was filtered using a syringe driven $0.22 \mu \mathrm{m}$ filter. The CSE was then adjusted to the desired OD using culture medium.

\section{Neutrophil isolation and culture}

Neutrophils were isolated from peripheral blood. Whole blood was mixed with $4 \%$ dextran (Sigma-Aldrich) in $1 \times$ phosphate buffered saline (PBS) and PBS at a ratio of 2:1:1 and the red blood cells were left to sediment on ice for 30 mins. The remaining supernatant was removed, layered over Ficoll-Paque (GE Healthcare, Buckinghamshire, UK) and centrifuged (400 $\mathrm{g}$ for $30 \mathrm{mins}$ at room temperature). The remaining red bloods cells were

Table 1 Brands of e-cig used in the study

\begin{tabular}{|c|c|c|c|}
\hline Brand & VIP (brand 1) & KIK (brand 2) & Puritane (brand 3) \\
\hline Model & $\begin{array}{l}\text { 1100mAh battery with } \\
\text { V5/CE5 clearomiser }\end{array}$ & $\begin{array}{l}900 \mathrm{mAh} \text { battery with } \\
\text { protank clearomiser }\end{array}$ & $\begin{array}{l}\text { Re-chargeable battery with } \\
\text { replacement cartomiser }\end{array}$ \\
\hline Flavour & USA Tobacco & USA Red & Original \\
\hline Nicotine Strength & $24 \mathrm{mg}$ & $24 \mathrm{mg}$ & $16 \mathrm{mg}$ \\
\hline
\end{tabular}

A summary of the brands, models of of e-cig and flavour of e-liquid used to generate e-cig vapour extract preparations for this study 
lysed by addition of sterile molecular grade water (Sigma-Aldrich) and the suspension was quenched by the addition of PBS. The suspension was then washed ( $400 \mathrm{~g}$ for $10 \mathrm{mins}$ at $4{ }^{\circ} \mathrm{C}$ ) and the granulocyte number was assessed by trypan blue exclusion. Granulocytes were re-suspended at a density of $1 \times 10^{6}$ per $\mathrm{ml}$ in supplemented RPMI-1640 media. The purity of the neutrophil preparation was approximately $95 \%$ (confirmed by Rapi-diff staining as previously described [32]). Cytospin preparations were air dried, fixed with methanol for ten minutes and stained with Rapi-diff according to manufacturer's instructions (Triangle, Skelmersdale, UK). A total of 200 cells per slide were counted and the proportion of neutrophils was calculated.

\section{Neutrophil shape change, viability and CD11b and CD66b expression}

Neutrophils were seeded at $5 \times 10^{5}$ cells per polypropylene tube and incubated with ECVE (0.001-0.1 OD) for 2, 4 or $6 \mathrm{~h}$ (shape change and $\mathrm{CD} 11 \mathrm{~b}$ and CD66b expression) or $6 \mathrm{~h}$ (viability) in a $5 \% \mathrm{CO}_{2}$ humidified atmosphere at $37{ }^{\circ} \mathrm{C}$. Cells were washed in PBS and prepared for flow cytometry. Details can be found in Additional file 3.

\section{MMP-9 and CXCL8 release}

Neutrophils were seeded at $1 \times 10^{5}$ cells per well in a flat bottomed 96-well plate and incubated with ECVE, CSE (0.001-0.1 OD) or acrolein (Sigma-Aldrich) for $6 \mathrm{~h}$ in a $5 \% \mathrm{CO}_{2}$ humidified atmosphere at $37{ }^{\circ} \mathrm{C}$. Supernatants were removed and analysed for MMP-9 and CXCL8 by enzyme linked immunosorbant assay (ELISA; R\&D Systems, Abingdon, UK) according to manufacturer's instructions. Supernatants were also used to measure MMP-9 activity by zymography and MMP-9 levels by western blot. Details can be found in Additional file 3.

For studies examining the effect of drug treatment on ECVE (0.003 OD) induced MMP-9 release, neutrophils were pre-treated with vehicle control (DMSO $0.005 \%$ ) the p38 MAPK inhibitor BIRB-796 (1 $\mu \mathrm{M}$; Sigma-Aldrich), the ERK $1 / 2$ inhibitor selumetinib $(1 \mu \mathrm{M}$; Stratech Scientific Ltd, Newmarket, UK) or dexamethasone ( $1 \mu \mathrm{M}$; Sigma-Aldrich) for $1 \mathrm{~h}$ prior to incubation with ECVE. MMP-9 was measured by ELISA.

\section{NE activity}

Neutrophils were seeded at $1.4 \times 10^{5}$ cells per well in a flat bottomed 96-well plate and incubated with ECVE (0.001-0.1 OD) for $6 \mathrm{~h}$ in a $5 \% \mathrm{CO}_{2}$ humidified atmosphere at $37{ }^{\circ} \mathrm{C}$. A rhodamine 110-based substrate (R6506 Invitrogen) was added $30 \mathrm{~min}$ prior to the endpoint after which fluorescence was measured using a FLUOstar omega plate reader set at excitation $485 \mathrm{~nm}$ emission 520 nm (BMG Labtech, Buckinghamshire, UK).

\section{Inflammatory pathway activation}

Neutrophils were seeded at $1 \times 10^{6}$ cells per well in a flat bottomed six-well plate and incubated with ECVE $(0.003$ and $0.01 \mathrm{OD}$ ) for 30 and $60 \mathrm{~min}$ in a $5 \% \mathrm{CO}_{2}$ humidified atmosphere at $37{ }^{\circ} \mathrm{C}$. Cells were then lysed in RIPA buffer [10 mM Tris-HCl, pH 7.4, 150 mM NaCl, 1 mM EDTA, 1 \% Nonidet P-40, $0.25 \%$ ] containing phosphatase (Sigma-Aldrich) and protease inhibitors (Calbiochem, Nottingham, UK) and samples were prepared for Western blot. Details can be found in Additional file 3.

\section{Ultra-high performance liquid chromatography mass spectrometry (UHPLC-MS) analysis}

UHPLC-MS was performed to analyse the chemical composition of e-liquid and ECVE. Details can be found in Additional file 3.

\section{Data analysis}

Statistical analyses were performed using GraphPad InStat software (GraphPad Software Inc., California, USA). Experiments with ECVE dose response curves were analysed using a one-way analysis of variance followed by a Dunnett's post-test.

\section{Results}

Neutrophil activation

Neutrophils $(n=3)$ were exposed to ECVE for up to $6 \mathrm{~h}$. ECVE increased neutrophil shape change and the mean fluorescence intensity of CD11b and CD66b expression compared to untreated neutrophils (Fig. 1 and Additional file 4); maximal changes were observed with 0.003 ECVE for $4 \mathrm{~h}(p<0.05)$. There was a bell shaped curve, as shape change, CD11b and CD66b expression levels were not increased at higher ECVE concentrations. Analysis using the percentage of $\mathrm{CD} 11 \mathrm{~b}$ positive neutrophils followed a similar trend but this did not reach statistical significance (Additional file 5), while there was no change for the percentage of CD66b positive neutrophils (Additional file 5). There was high baseline expression of both of these markers when using percentage expression analysis. Following ECVE treatment for $6 \mathrm{~h}$, the percentage of neutrophils undergoing early apoptosis or necrosis were not increased compared to untreated neutrophils (Additional file 6).

\section{Protease and CXCL8 secretion}

Neutrophils $(n=10)$ exposed to ECVE secreted increased quantities of MMP-9 and CXCL8 with maximal release observed at 0.003 ECVE $(p<0.01$ and $p<0.05$ respectively; Fig. 2a and d). The secretion of MMP-9 and CXCL8 showed a bell shaped curve, with reduced secretion at higher concentrations. We confirmed that there was no contamination of the ECVE by the apparatus, by preparing 


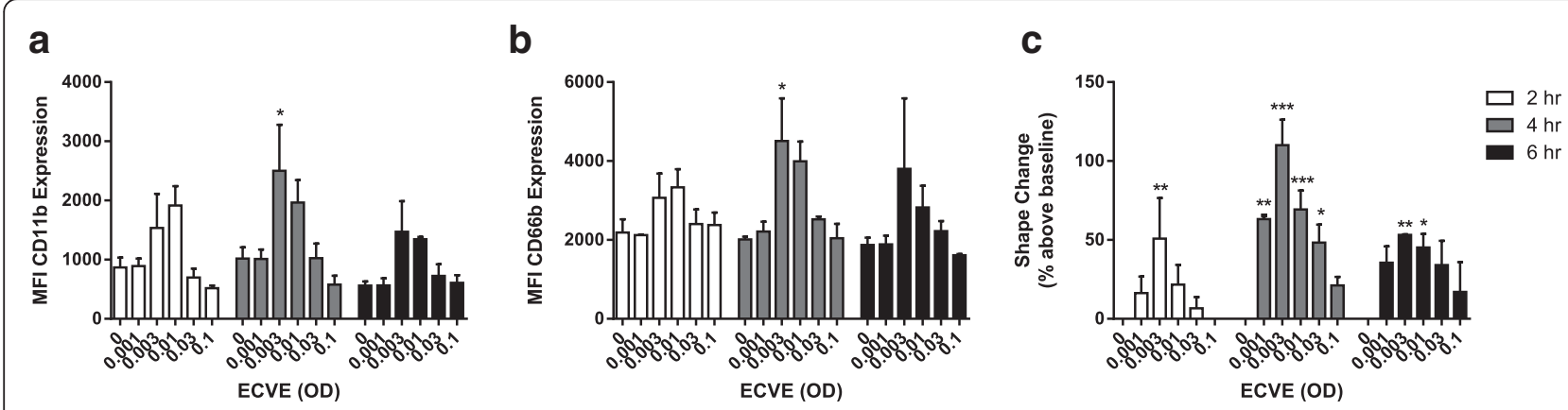

Fig. 1 E-cig induced neutrophil activation. Neutrophils from three healthy subjects were exposed to ECVE (0.001-0.1 OD) for 2, 4 or 6 h (white, grey and black bars respectively). Neutrophils were analysed for CD11b (a) and CD66b (b) expression, and shape change (c) by flow cytometry. Data presented as mean \pm SEM where ${ }^{*}{ }^{* *}$ and ${ }^{* * *}=$ significant increase compared to unstimulated control $(p<0.05, p<0.01$, and $p<0.001$ respectively)

a "mock" ECVE, bubbling air through the culture medium (Additional file 7).

Gelatinase activity in neutrophil supernatants $(n=3)$ was increased following exposure to 0.003 ECVE (Fig. 2b). Activity was observed in the $90-150 \mathrm{kDa}$ range, consistent with different forms of MMP-9. Western blot analysis confirmed MMP-9 was present within the 90-150 kDa range (Fig. 2b), and densitometry confirmed a significant increase in MMP-9 secretion upon exposure to 0.003 ECVE compared to untreated neutrophils (Fig. 2c).

\section{a}

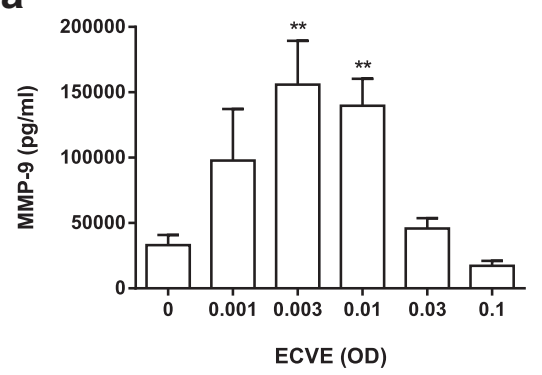

b

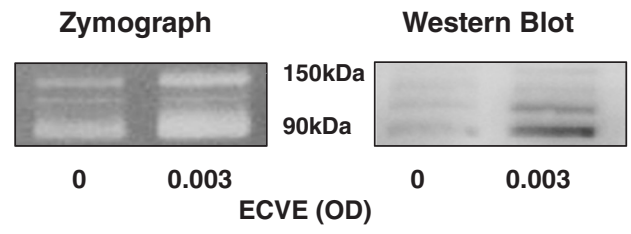

C

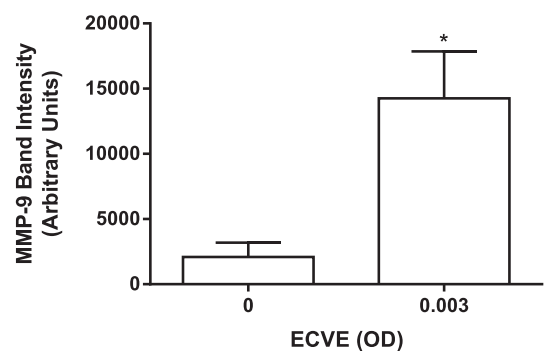

d

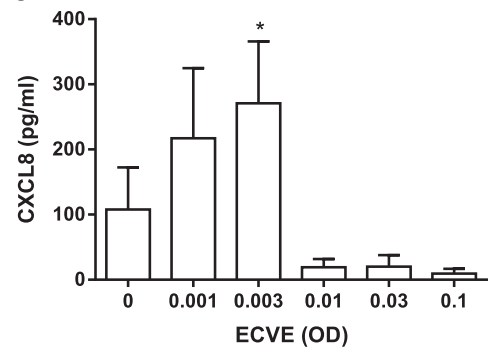

e

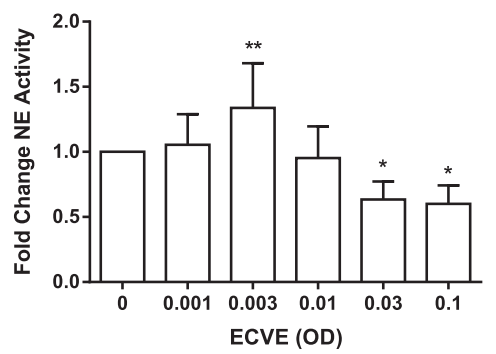

Fig. 2 E-cig induced protease and CXCL8 release. Neutrophils from healthy subjects were exposed to ECVE (0.001-0.1 OD) for 6 h and supernatants were analysed for MMP-9 $(\mathbf{a})$ and CXCL8 $(\mathbf{d})$ release by ELISA $(n=10)$ and NE activity by florescence $(\mathbf{e} ; n=6)$. Culture supernatants from healthy subjects (0.003 ECVE) were also analysed for MMP-9 activity by zymography and MMP-9 expression by western blot (representative image; (b)). Western blots were analysed by densitometry $(\mathbf{c})$. Data presented as mean \pm SEM where ${ }^{*},{ }^{*}$ and ${ }^{* * *}=$ significant change compared to unstimulated control $(p<0.05, p<0.01$, and $p<0.001$ respectively) 
NE activity was significantly increased in the supernatants of neutrophils $(N=6)$ exposed to 0.003 ECVE compared to untreated neutrophils $(p<0.01$; Fig. $2 \mathrm{e})$. A bell shaped curve was again observed, with a significant reduction in NE activity at 0.03 and $0.1 \mathrm{ECVE}(p<0.05)$.

\section{Cell signalling pathway activation}

Cigarette smoke has previously been shown to activate the p38 MAPK and ERK pathways in neutrophils [33-35]. In addition, nicotine has been shown to activate the NF- $\mathrm{kB}$ pathway ([36]. We therefore examined the effect of ECVE on the activation of these pathways.

Western blot analysis demonstrated a significant increase in p38 MAPK phosphorylation at 0.01 ECVE $(p<0.05)$ and a numerical increase at 0.003 compared to unstimulated controls $(n=5$; Fig. 3$)$. There was a numerical increase in ERK $1 / 2$ phosphorylation that did not reach statistical significance. There were no changes in the phosphorylation of the p65 subunit of nuclear factor kappa-light-chain-enhancer of activated B cells (NF-kB).

We further investigated the involvement of p38 MAPK in ECVE induced neutrophil activation by studying the effect of a p38 MAPK inhibitor, BIRB-796, on MMP-9 release in response to $\operatorname{ECVE~}(n=6)$. We used 0.003 ECVE as this concentration caused the greatest secretion of MMP-9 (Fig. 2). Pre-treatment of neutrophils with BIRB-796 $(1 \mu \mathrm{M})$ for $1 \mathrm{~h}$ prior to incubation with 0.003 ECVE for $6 \mathrm{~h}$ significantly reduced MMP-9 release by $62 \%$ (Fig. 4). The ERK inhibitor, selumetinib $(1 \mu \mathrm{M})$, and the corticosteroid dexamethasone $(1 \mu \mathrm{M})$ had no effect.

\section{E-Cig brand and CSE comparisons}

Nicotine has previously been shown to induce CXCL8 release from neutrophils [36]. We therefore sought to evaluate the effects of different e-cig brands with and without nicotine on MMP-9 and CXCL8 release $(n=10$; Figs. 5 and 6).

All the brands increased MMP-9 and CXCL8 secretion (although the increase in MMP-9 secretion after exposure to KIK $24 \mathrm{mg}$ did not reach statistical significance), with bell shaped curves observed for most brands. The ECVE concentrations that caused the greatest inductions of MMP-9 and CXCL8 secretion varied greatly between brands. CSE caused significant increases in MMP-9 secretion at 0.03 and 0.1 OD and CXCL8 secretion at 0.01 and $0.03 \mathrm{OD}$. The effects of the different e-cig brands were often similar to, or greater than, CSE.

\section{UHPLC-MS analysis}

The labelling stating the constituents of brand $1(24 \mathrm{mg})$ e-cig liquid was as follows: nicotine, water, propylene glycol, glycerol and flavours. We investigated the constituents of e-cig liquid using UHPLC-MS. Chemicals were putatively identified based on molecular weight, with other constituents unidentified. Their chemical class and potential hazards are documented within Table 2, along with the associated chemical entities of biological interest (ChEBI) code [37], which enables cross-referencing of chemicals for biological relevance. Cyclohexane, isoquinoline, diglyme and skatole were identified.

A similar analysis was carried out on ECVE prepared using brand 1 ( $24 \mathrm{mg}$ ) e-cig liquid. Over 120 chemicals

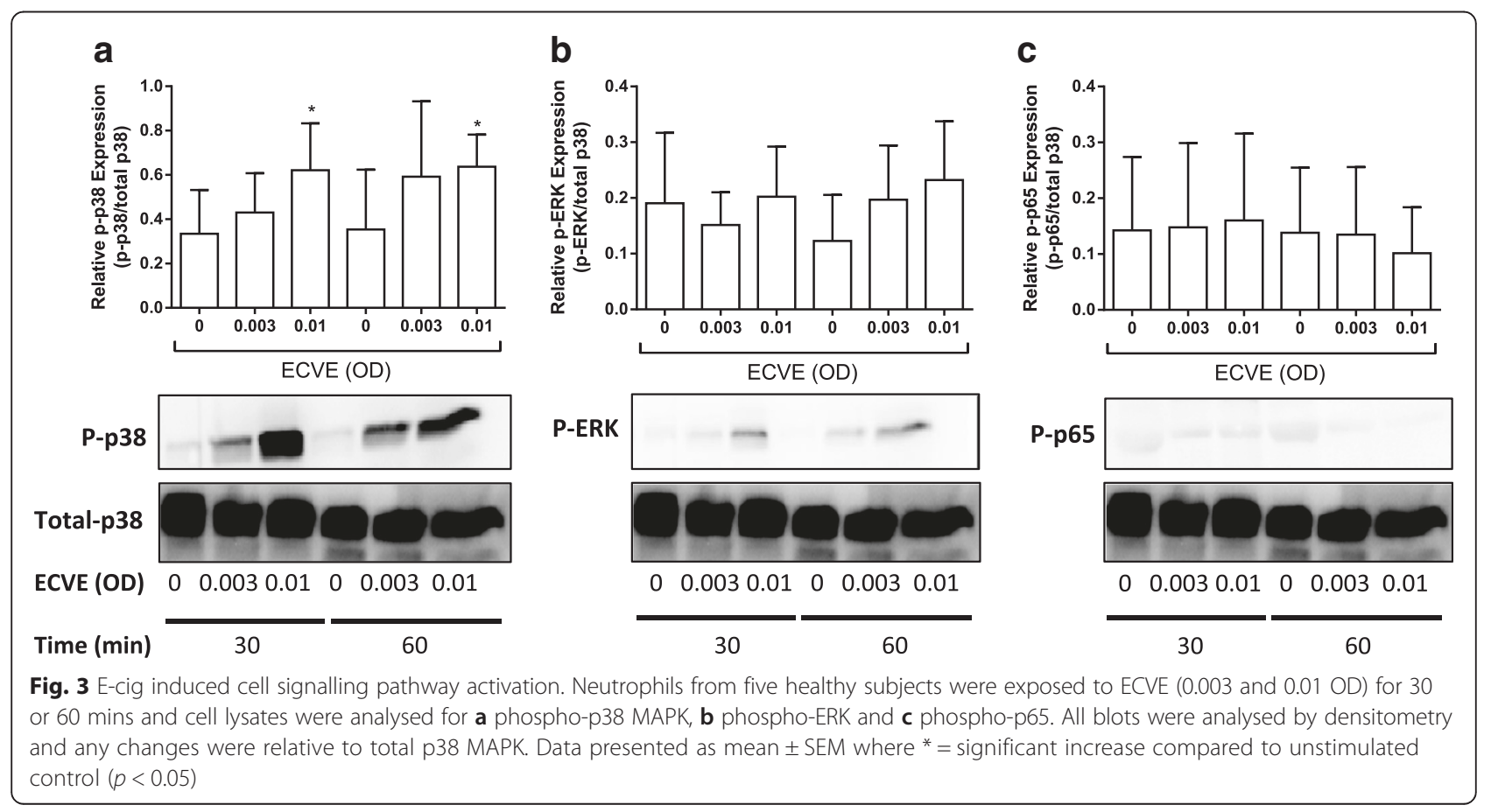




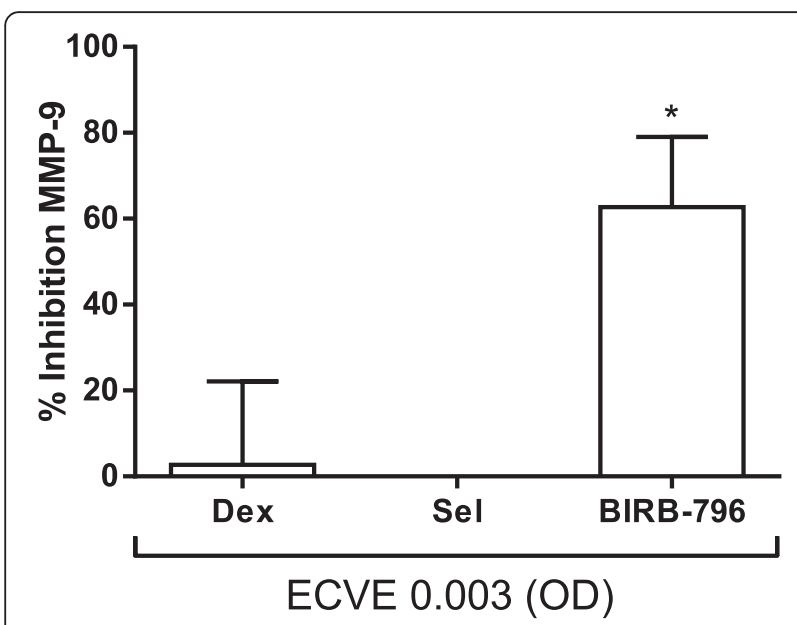

Fig. 4 Inhibition of e-cig induced MMP-9 release. Neutrophils from six healthy subjects were pre-incubated with dexamethasone (dex; $1 \mu \mathrm{M})$, selumetinib (Sel; $1 \mu \mathrm{M})$ or BIRB-796 (1 $\mu \mathrm{M})$ for $1 \mathrm{~h}$ prior to ECVE (0.003) exposure for $6 \mathrm{~h}$. Supernatants were analysed for MMP-9 by ELISA. Data presented as mean \pm SEM where ${ }^{*}=$ significant increase compared to unstimulated control $(p<0.05)$

were putatively identified (Table 3 ), with extra constituents potentially coming from e-cig liquid vaporisation byproducts. Known harmful chemicals including acrolein, propanal, styrene and the carcinogens 2, 3-benzofuran and allylthiourea were putatively identified.

We also performed pure standard analysis of acrolein (Additional file 8). Inference of median ECVE ion count levels of acrolein from the standard analysis indicates a level of $4.81 \mu \mathrm{M}$ present in ECVE.

\section{Effect of acrolein on neutrophil response}

The presence of high acrolein levels $(4.81 \mu \mathrm{M})$ in ECVE prepared with brand $1(24 \mathrm{mg})$ led us to examine the effects of acrolein $(0.1-1 \mu \mathrm{M})$ on the neutrophil inflammatory response from six healthy subjects. Acrolein caused a significant increase in MMP-9 release at a concentration of $1 \mu \mathrm{M}$ (Fig. 7).

\section{Discussion}

ECVE causes activation of human neutrophils. ECVE increased neutrophil CD11b and CD66b expression, and induced neutrophil shape change; these are markers of neutrophil activation. Furthermore, ECVE increased MMP-9 and CXCL8 secretion by neutrophils, and upregulated MMP-9 and NE activity. These pro-inflammatory changes were accompanied by p38 MAPK activation. Mass spectroscopy analysis of e-cig liquid and ECVE confirmed the presence of various compounds capable of causing harm to humans. These findings challenge the perception that e-cigs are safe.

It is known that acute exposure to cigarette smoke extract increases CXCL8 and MMP-9 secretion from alveolar macrophages and neutrophils, $[7,38]$ and NE secretion from neutrophils $[8,16]$. Here we show similar findings for the effects of ECVE on neutrophils. Neutrophil activation is a characteristic feature of COPD; indeed,

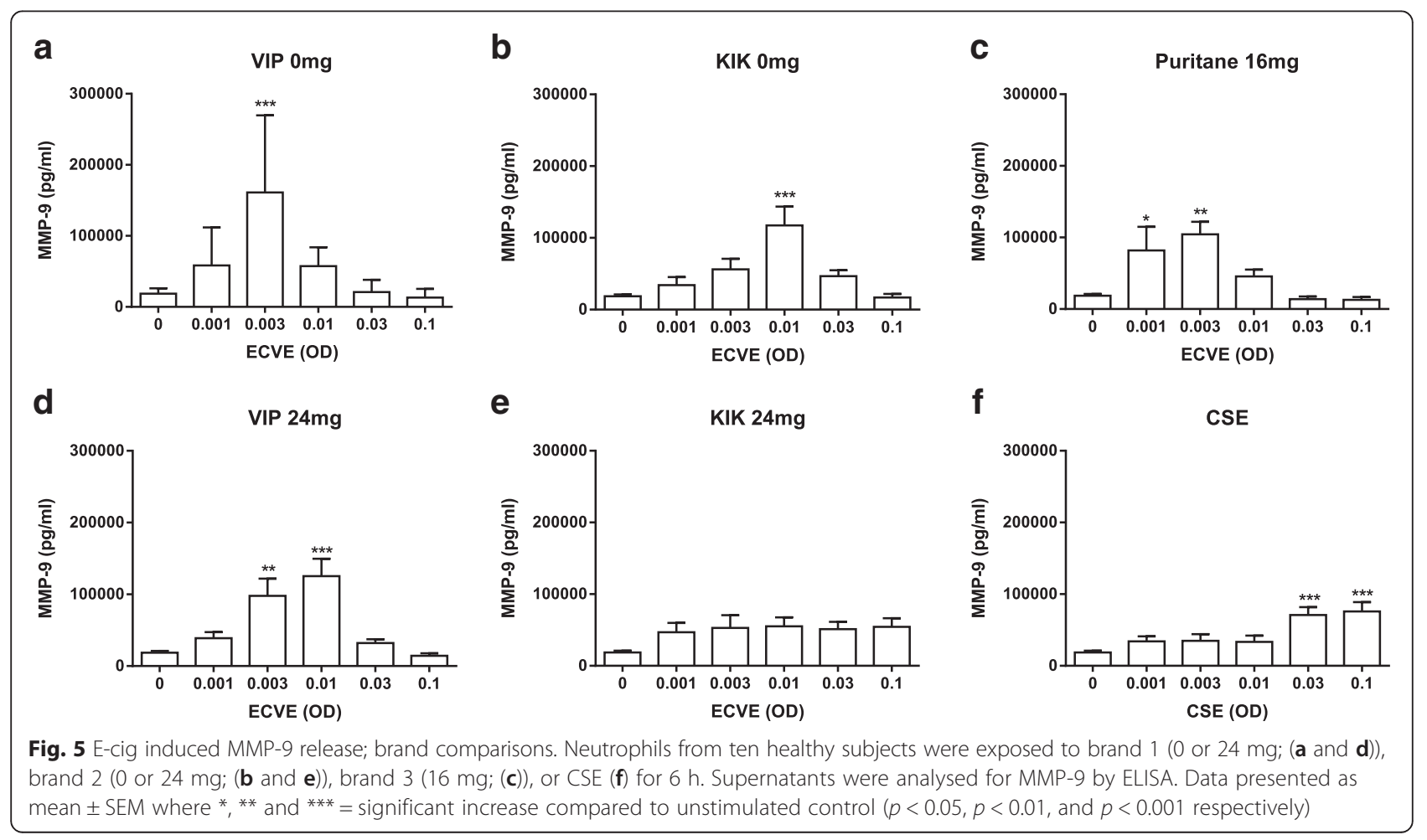




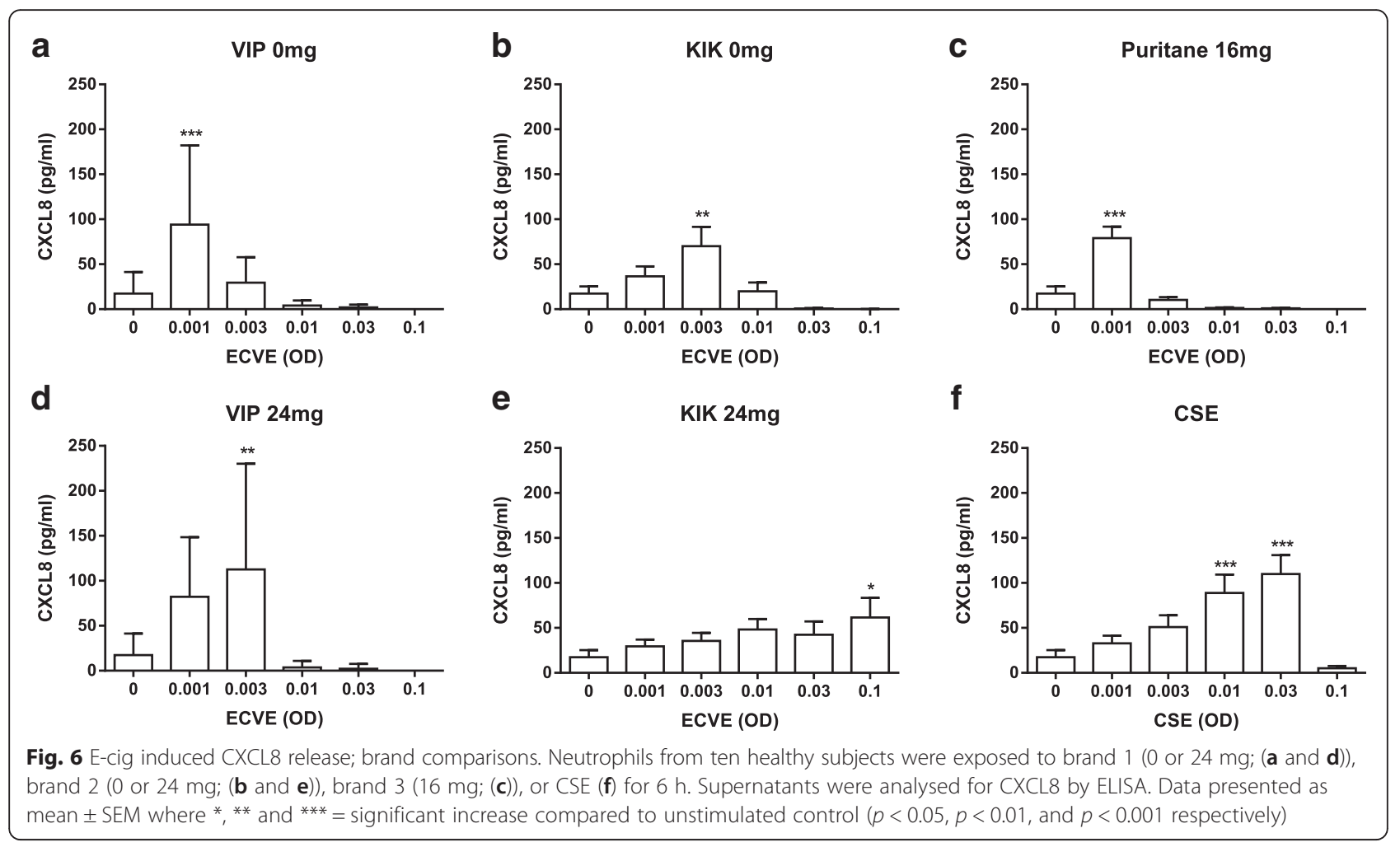

Table 2 Chemicals putatively identified in brand 1 (24 mg) e-liquid

\begin{tabular}{|c|c|c|c|c|}
\hline Chemical & Hazard statement & Hazard class & Type of compound & ChEBI code \\
\hline Anabasine;nicotine;TC-2403-12"; & $\mathrm{H} 301 / \mathrm{H} 310 / \mathrm{H} 400 / \mathrm{H} 411$ & Toxic/Dangerous & Active drug & 18723 \\
\hline Glycerol & N/A & N/A & Solubilizing agent & 17754 \\
\hline 1,4-Cyclohexanedione;2-hexenedial & S22/S23/S24/S25 & Irritant & Cyclohexane analogue & 28286; N/A \\
\hline Skatole;tolylacetonitrile" & H315/H319/H335/H411 & Warning/Senatizer & Coal tar extract - flavour & $9171 ; 27982$ \\
\hline 1-Methoxy-2-(2-methoxyethoxy)ethane & H226/H360FD & Health Hazard/Flammable & $\begin{array}{l}\text { Diglyme - toxic digylcerol } \\
\text { breakdown product }\end{array}$ & 46784 \\
\hline Nicotyrine & N/A & Toxic/Dangerous & Nicotine breakdown product & 7564 \\
\hline $\begin{array}{l}\text { methyl 10,12-dihydroperoxy-8E, } \\
\text { 13E,15Z-octadecatrienoate }\end{array}$ & N/A & N/A & Fatty acid & N/A \\
\hline Isoquinoline;quinoline;"; & H302/H310/H315 & Acutely Toxic/Dangerous & Bicyclic pyridine analogue & 16092; 17362 \\
\hline 8-hydroxy-nonanoic acid & N/A & N/A & Hydroxylated fatty acid & N/A \\
\hline 2,3,5-Trihydroxytoluene & N/A & N/A & N/A & 17185 \\
\hline Monocyclic botryococcane;" & N/A & N/A & Isoprenyl-lipid & N/A \\
\hline $\begin{array}{l}\text { 1-Methoxy-2-(2-methoxyethoxy)ethane; } \\
\text { "2-methylpentane-1,2,4-triol";"; }\end{array}$ & $\mathrm{H} 226 / \mathrm{H} 360 \mathrm{FD}$ & Health Hazard/Flammable & Diglyme - toxic digylcerol & 46784 \\
\hline 2,3-Dihydroxyvaleric acid & N/A & N/A & Pentose-sugar & N/A \\
\hline Pentaethylene glycol monodecyl ether;"; & N/A & N/A & Detergent & N/A \\
\hline Unknown lipid & N/A & N/A & N/A & N/A \\
\hline Unknown lipid & N/A & N/A & N/A & N/A \\
\hline Unknown lipid & N/A & N/A & Sphingolipid & N/A \\
\hline $\begin{array}{l}\text { Isopentenyladenine-7-N-glucoside;"; } \\
\text { 12-oxo-20-trihydroxy-leukotriene B4;" }\end{array}$ & N/A & N/A & N/A & $74321 ; \mathrm{N} / \mathrm{A}$ \\
\hline Ethyl-trimethyl-silane & N/A & N/A & $\begin{array}{l}\text { Potential silica breakdown } \\
\text { product from wick }\end{array}$ & 48788 \\
\hline
\end{tabular}


Table 3 Chemicals putatively identified in ECVE prepared with brand 1 (24 mg)

\begin{tabular}{|c|c|c|c|c|}
\hline Chemical & Hazard statement & Hazard class & Type of compound & ChEB| \\
\hline Nicotine & $\mathrm{H} 301 / \mathrm{H} 310 / \mathrm{H} 400 / \mathrm{H} 411$ & Toxic/Dangerous & Active drug & 18723 \\
\hline Glycerol & N/A & N/A & Solubilizing agent & 17754 \\
\hline gamma-hexenoic acid & H314/R34 & Danger/Corrosive & Unsaturated fatty acid & 38355 \\
\hline 2-methyl valeric acid & H314/R34 & Danger/Corrosive & Saturated fatty acid & N/A \\
\hline 2-Methylpentane-1,2,4-Triol & N/A & N/A & Saturated polyol & N/A \\
\hline 4-Aminohydrocinnamic Acid & H315/H319/H335 & Warning//rritant & saturated carboxylic acid & N/A \\
\hline 5-oxo-7-octenoic acid & N/A & N/A & Branched fatty acid & N/A \\
\hline Piperidine & $\mathrm{H} 225 / \mathrm{H} 311 / \mathrm{H} 331 / \mathrm{H} 314$ & Corrosive/Highly Flammable/Toxic & Organic Heterocyclic & 18049 \\
\hline Propanal & H225/H302/H332/H315/H318/H335 & Highly Flammable/Harmful//rritant & $\begin{array}{l}\text { Small organic (possible glycerol } \\
\text { breakdown) }\end{array}$ & 17153 \\
\hline (2R,3S)-2,3-Dimethylmalate & N/A & Natural & Possible cellular metabolite & 57422 \\
\hline 2,3-Benzofuran & $\mathrm{H} 226 / \mathrm{H} 351$ & Flammable/Carcinogen & Heterocyclic & 36790 \\
\hline 4-(3-pyridyl)-butanoate & N/A & N/A & $\begin{array}{l}\text { Nitrosonornicotine metabolite } \\
\text { derived from tobacco smoke. }\end{array}$ & 66942 \\
\hline N-Nitroso-1,3-thiazolidine & N/A & N/A & Polar small molecule & 7329 \\
\hline allylthiourea & H301 & Toxic/Carcinogen & Polar small molecule & 74079 \\
\hline 2-methyl-1-butanol & N/A & N/A & Polar small molecule & 50625 \\
\hline Acrolein & $\mathrm{H} 225 / \mathrm{H} 301 / \mathrm{H} 311 / \mathrm{H} 314 / \mathrm{H} 330 / \mathrm{H} 400$ & Poisonous/Irritant/Flammable & Glycerol breakdown product & 15368 \\
\hline Styrene & H226/H315/H319/H332 & Harmful/Irritant & Unsaturated aromatic & 27452 \\
\hline
\end{tabular}

COPD is often referred to as a "neutrophilic lung disease". We demonstrate that e-cigs cause neutrophil activation, which raises concerns about the safety of these devices.

A proposed advantage of e-cigs is that they lack the carcinogens within the tar present in cigarettes. However, e-cigs may still contain compounds with carcinogenic properties [5]. We now show that e-cigs also contain a variety of other compounds, including carcinogens. These include the saturated aldehyde propanal, the carcinogens

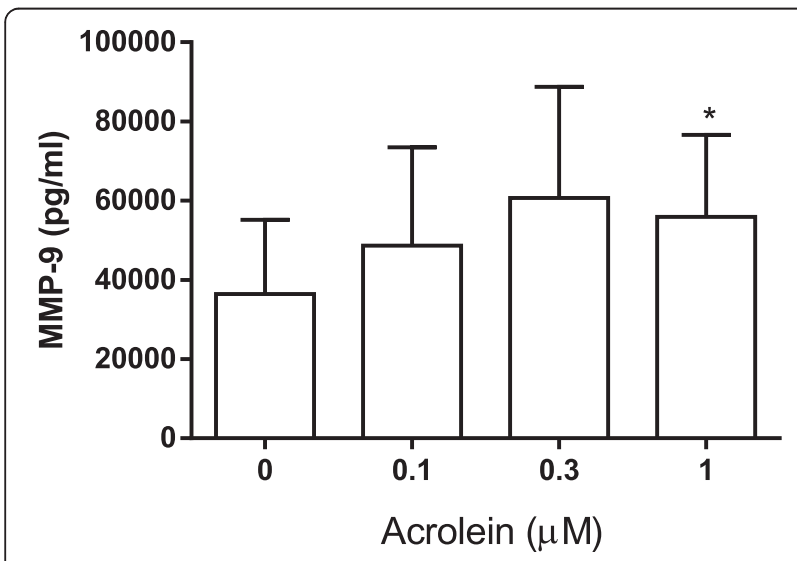

Fig. 7 Acrolein induced MMP-9 release. Neutrophils from six healthy subjects were exposed to acrolein $(0.1-1 \mu \mathrm{M})$ for $6 \mathrm{~h}$. Supernatants were analysed for MMP-9 by ELISA. Data presented as mean \pm SEM where $^{*}=$ significant increase compared to unstimulated control $(p<0.05)$
2, 3-benzofuran and allylthiourea and the respiratory toxin styrene.

We observed acrolein in the vapour extract but not the e-liquid itself. Acrolein can be produced by heating glycerol [39], and our observations are likely attributable to heating glycerol contained in e-liquid. Acrolein is also a constituent of tobacco smoke. Acrolein induces inflammatory responses from macrophages and epithelial cells $[40,41]$. We now show that acrolein induces MMP-9 release from neutrophils within the concentration found in ECVE. This confirms that acrolein is one potential cause of the pro-inflammatory effects of e-cig vapour. We have identified a number of chemicals present in ECVE using UHPLC-MS, and the potential for each of these to cause inflammation or other toxic effects can now be investigated. Styrene has previously been linked to airway inflammation present in people with occupational asthma [42] and has also been shown to induce monocyte chemotactic protein-1 (MCP-1) and CXCL8 release from an alveolar epithelial cell line $[43,44]$. In addition, propanal has been shown to increase gene expression of several pro-inflammatory cytokines and chemokines including IL-6 and CXCL8 in an alveolar epithelial cell line [45].

We observed a bell shaped curve for the effect of ECVE in all experiments of neutrophil function. This was not due to a reduction in neutrophil viability. There are other examples of bell shaped curves for neutrophil biology, including CXCL8 induced chemotaxis, substance 
$\mathrm{P}$ induced adhesion and Staphylococcus aureus induced CD11b, CD18 and CD66b expression [46-48]. It has been suggested that the bell shaped curve for neutrophil function arises due to negative feedback mechanisms. For example, CXCL8 causes a dose dependant reduction in chemokine C-X-C motif receptor (CXCR) 1 and CXCR2 expression. Reducing the expression of these chemotactic receptors regulates neutrophil inflammatory responses and arrests these cells at the site of inflammation where chemokine concentrations are highest.

CD66b cross-linking between neutrophils induces CXCL8 release [49]. When neutrophils are exposed to ECVE, CD66b expression may be driving the release of inflammatory mediators through neutrophil cross-linkage. However, at higher ECVE concentrations this CD66b response diminishes. This may regulate neutrophil responses to avoid excessive inflammation, similar to the suggestion of Schmidt et al in the case of neutrophils exposed to bacteria [47]. However, neutrophils artificially arrested in this state are less likely to exert their protective innate immune functions and therefore the host may be more susceptible to bacterial infection.

The analysis of CD11b and CD66b principally used median fluorescence intensity (MFI) by flow cytometry to provide a sensitive overall quantification of expression in all neutrophils. The analysis of cell percentages is a commonly used alternative, but in this case there were high baseline expression levels which made it difficult to observe any increase with ECVE.

MMP-9 levels are increased in the lungs of smokers [50] and there is a positive correlation between MMP-9 levels, sputum neutrophils and COPD severity [17, 18, 51]. Similarly, NE levels are increased in the lungs of cigarette smokers and NE activity is positively correlated with COPD severity [19, 52]. MMP-9 and NE contribute to emphysema through the destruction of the lung parenchyma. Our results suggest that e-cig use increases the ability of human neutrophils to secrete activated MMP-9 and NE, which may contribute to lung parenchymal destruction.

$\mathrm{NE}$ degrades the antimicrobial peptide, short palate lung and nasal epithelium clone 1 (SPLUNC1) [53]. E-liquid inhibits SPLUNC1 expression in human airway epithelial cells [10]. Both direct and indirect reductions in SPLUNC1 levels by e-cigs in addition to reduced bacterial phagocytic activity of e-cig exposed alveolar macrophages [54], may result in a susceptibility to bacterial infections in e-cig users. Mice exposed to e-cig vapour have been shown to be more susceptible to viral and bacterial infections [54]. This is reminiscent of the effects of tobacco smoking.

ECVE caused activation of the p38 MAPK pathway, akin to tobacco smoke exposed alveolar macrophages [7, 41]. P38 MAPK activation promotes inflammation and activated p38 MAPK is increased in COPD lungs $[52,55]$.
We showed activation of the p38 MAPK pathway at 0.01 ECVE. The results at 0.003 ECVE were not statistically significant, despite clear activation in some of the experiments performed (Fig. 3). The 0.003 ECVE concentration generally caused the greatest increases in cell activation (Figs. 1 and 2), although there was evidence that 0.01 ECVE caused significant MMP-9 release and neutrophil shape change. The p38 MAPK inhibitor BIRB-796 suppressed 0.003 ECVE induced MMP-9 release by approximately $60 \%$, confirming the role of p38 MAPK signalling in the neutrophil response to ECVE. However, approximately $40 \%$ of ECVE induced MMP-9 release was insensitive to p38 MAPK inhibition suggesting ECVE can also induce MMP-9 release by p38 MAPK independent mechanisms.

The corticosteroid dexamethasone had no effect on ECVE induced MMP-9 release. This may be relevant to COPD patients using e-cigs and corticosteroids; these drugs do not appear to suppress neutrophil activation caused by e-cigs.

E-cig induced MMP-9 and CXCL8 release was demonstrated using different brands of e-cigs with and without nicotine. This demonstrates that the pro-inflammatory effects are not restricted to one brand and are observed using preparations with and without nicotine. There were considerable differences in the magnitude of effects between the brands, and the shape of the dose response curves, which are likely attributable to differences in chemical composition. The increased flow rate used to produce ECVE with brand 3 did not result in an increased magnitude of effect when compared to the other brands. Our comparisons with cigarette smoke provide important insights; MMP-9 and CXCL8 release caused by different e-cig brands were often similar to, or in excess of, the CSE response.

Nicotine has previously been shown to induce CXCL8 release from neutrophils [36]. We observed increased release of MMP-9 and CXCL8 upon exposure to ECVE with and without nicotine, suggesting that these effects are dependent on other pro-inflammatory constituents.

The primary aim of this paper was to examine the potential of e-cigs to cause harm. We used neutrophils from healthy never smokers to avoid any confounding variables due to chronic exposure to tobacco cigarettes or the presence of chronic inflammation. Nevertheless, future studies should examine the effect of e-cigs on immune cells isolated from COPD patients, particularly those involved in the pathogenesis of COPD, including neutrophils, alveolar macrophages and CD8 T-cells. This will help us to understand the impact of e-cigs on patients with established chronic inflammation.

ECVE causes rapid activation of neutrophils which continues up to $6 \mathrm{~h}$. Although we do not know how repeated exposure to e-cigs will affect neutrophils, the 
acute effects reported here are similar to those by cigarette smoke extract. This suggests that chronic exposure to e-cigs may induce similar chronic inflammatory changes reported in cigarette smokers.

\section{Conclusions}

We have shown that ECVE causes pro-inflammatory responses from human neutrophils. E-cigs are often used to avoid the unwanted effects of conventional cigarettes, such as causing pulmonary inflammation. Our results raise concerns over the safety of e-cig use.

\section{Additional files}

Additional file 1: Demographics of the study population. (DOCX $13 \mathrm{~kb}$ ) Additional file 2: The absorbance spectrum ECVE preparations. The optical density of e-cig vapour extract preparations for VIP $0 \mathrm{mg}$ (blue), VIP 24 mg (red), KIK 0 mg (green), KIK 24 mg (yellow), Puritane (orange) and cigarette smoke extract (CSE; pink) were measured from 200-1000 nM. (PPTX $93 \mathrm{~kb}$ )

Additional file 3: Supplementary methods. (DOCX $28 \mathrm{~kb}$ )

Additional file 4: Raw flow cytometry data. Flow cytometry plots of ECVE exposed neutrophils examined for CD11b (left panel), CD66b (middle panel) and shape change (right panel). The light grey peaks indicate the unstained control and the dark grey peaks indicate the antibody staining. (PPTX $274 \mathrm{~kb}$ )

Additional file 5: The percentage of CD11b and CD66b positive neutrophils. Neutrophils from three healthy subjects were exposed to ECVE (0.001-0.1 OD) for 2, 4 or $6 \mathrm{~h}$ (white, grey and black bars respectively). Neutrophils were analysed for CD11b (a) and CD66b (b) expression by flow cytometry. Data presented as mean \pm SEM (PPTX $178 \mathrm{~kb}$ )

Additional file 6: Effect of e-cig exposure on neutrophil viability. Neutrophils from three healthy subjects were exposed to ECVE (0.001-0.1 OD) for $6 \mathrm{~h}$ and the percentage of cells undergoing early apoptosis (a) or late apoptosis (b) was quantified by flow cytometry. Data presented as mean \pm SEM. (PPTX $133 \mathrm{~kb}$ )

Additional file 7: A comparison between a mock ECVE preparation and media alone. Neutrophils from 10 healthy subjects were exposed to a mock ECVE preparation or media alone for $6 \mathrm{~h}$ and supernatants were analysed for MMP-9 release by ELISA. (PPTX $62 \mathrm{~kb}$ )

Additional file 8: The concentration of selected harmful chemicals contained within ECVE. The concentration of acrolein was estimated based upon the median ion count levels calculated from pure standard analysis. (PPTX $46 \mathrm{~kb}$ )

\section{Competing interests}

AH, NJWR, JD, DKT, SJF and RG have no conflicts of interests to disclose. DS has received sponsorship to attend international meetings, honoraria for lecturing or attending advisory boards and research grants from various pharmaceutical companies including Almirall, AstraZeneca, Boehringer Ingelheim, Chiesi, Genentech, GlaxoSmithKline, Glenmark, Johnson and Johnson, Merck, NAPP, Novartis, Pfizer, Skypharma, Takeda, Teva, Therevance and Verona. The authors declare that they have no competing interests.

\section{Authors' contributions}

All authors were involved in study conception and design, data interpretation and manuscript revision. AH conducted cell culture work and drafted the manuscript. NJWR conducted chemical analysis and drafted the manuscript. JD conducted flow cytometry. DKT conducted chemical analysis. DS was a major contributor to manuscript preparation. AH is the guarantor of the content of the manuscript, including data and analysis. All authors read and approved the final manuscript.

\section{Acknowledgements}

This work was supported by the North West Lung Centre Charity, Manchester. This report is independent research supported by National Institute for Health Research South Manchester Respiratory and Allergy Clinical Research Facility at University Hospital of South Manchester NHS Foundation Trust. The views expressed in this publication are those of the authors and not necessarily those of the NHS, the National Institute for Health Research or the Department of Health. Many thanks to Dr Simon Lea for help with flow cytometry analysis.

\section{Author details}

${ }^{1}$ Manchester Academic Health and Science Centre, University Hospital of South Manchester Foundation Trust, Centre for Respiratory and Allergy Medicine, Institute of Inflammation and Repair, Faculty of Medical and Human Sciences, The University of Manchester, Manchester, UK. ${ }^{2}$ Manchester Institute of Biotechnology, School of Chemistry, The University of Manchester, Manchester, UK.

Received: 21 October 2015 Accepted: 27 February 2016

Published online: 17 May 2016

\section{References}

1. Euromonitor International: Vapor devices and e-Cigarettes in the Global Tobacoo Market. 2015. http://blog.euromonitor.com/2015/06/vapor-devicesand-e-cigarettes-in-the-global-tobacco-market.html. Accessed 1 Sept 2015.

2. Etter J-F, Bullen C. Electronic cigarette: users profile, utilization, satisfaction and perceived efficacy. Addiction. 2011;106:2017-28.

3. Summary of results: laboratory analysis of electronic cigarettes conducted By FDA [http://www.fda.gov/NewsEvents/PublicHealthFocus/ucm173146.htm]. Accessed 1 Sept 2015.

4. Trehy ML, Ye W, Hadwiger ME, Moore TW, Allgire JF, Woodruff JT, Ahadi SS, Black JC, Westenberger BJ. Analysis of electronic cigarette cartridges, refill solutions, and smoke for nicotine and nicotine related impurities. J Liq Chromatogr Relat Technol. 2011;34:1442-58.

5. Jensen RP, Luo W, Pankow JF, Strongin RM, Peyton DH. Hidden formaldehyde in e-cigarette aerosols. N Engl J Med. 2015;372:392-4.

6. Mio T, Romberger DJ, Thompson AB, Robbins RA, Heires A, Rennard SI. Cigarette smoke induces interleukin-8 release from human bronchial epithelial cells. Am J Respir Crit Care Med. 1997;155:1770-6.

7. Metcalfe HJ, Lea S, Hughes D, Khalaf R, Abbott-Banner K, Singh D. Effects of cigarette smoke on Toll-like receptor (TLR) activation of chronic obstructive pulmonary disease (COPD) macrophages. Clin Exp Immunol. 2014;176:461-72.

8. Overbeek SA, Braber S, Koelink PJ, Henricks PA, Mortaz E, LoTam Loi AT, Jackson PL, Garssen J, Wagenaar GT, Timens W, et al. Cigarette smoke-induced collagen destruction; key to chronic neutrophilic airway inflammation? PLoS One. 2013;8:e55612.

9. Cervellati F, Muresan XM, Sticozzi C, Gambari R, Montagner G, Forman HJ, Torricelli C, Maioli E, Valacchi G. Comparative effects between electronic and cigarette smoke in human keratinocytes and epithelial lung cells. Toxicol in Vitro. 2014;28:999-1005.

10. Wu Q, Jiang $D$, Minor $M, C h u$ HW. Electronic cigarette liquid increases inflammation and virus infection in primary human airway epithelial cells. PLoS One. 2014:9:e108342.

11. Hogg JC, Chu F, Utokaparch S, Woods R, Elliott WM, Buzatu L, Cherniack RM Rogers RM, Sciurba FC, Coxson HO, Pare PD. The nature of small-airway obstruction in chronic obstructive pulmonary disease. N Engl J Med. 2004; 350:2645-53.

12. Pesci A, Majori M, Cuomo A, Borciani N, Bertacco S, Cacciani G, Gabrielli M. Neutrophils infiltrating bronchial epithelium in chronic obstructive pulmonary disease. Respir Med. 1998;92:863-70.

13. de Boer WI, Sont JK, van Schadewijk A, Stolk J, van Krieken JH, Hiemstra PS. Monocyte chemoattractant protein 1 , interleukin 8 , and chronic airways inflammation in COPD. J Pathol. 2000;190:619-26.

14. Keatings VM, Collins PD, Scott DM, Barnes PJ. Differences in interleukin-8 and tumor necrosis factor-alpha in induced sputum from patients with chronic obstructive pulmonary disease or asthma. Am J Respir Crit Care Med. 1996;153:530-4.

15. Sharafkhaneh A, Hanania NA, Kim V. Pathogenesis of emphysema: from the bench to the bedside. Proc Am Thorac Soc. 2008;5:475-7.

16. Guzik K, Skret J, Smagur J, Bzowska M, Gajkowska B, Scott DA, Potempa JS. Cigarette smoke-exposed neutrophils die unconventionally but are rapidly phagocytosed by macrophages. Cell Death Dis. 2011;2:e131. 
17. Vlahos R, Wark PA, Anderson GP, Bozinovski S. Glucocorticosteroids differentially regulate MMP-9 and neutrophil elastase in COPD. PLoS One. 2012;7:e33277.

18. Beeh KM, Beier J, Kornmann O, Buhl R. Sputum matrix metalloproteinase-9, tissue inhibitor of metalloprotinease-1, and their molar ratio in patients with chronic obstructive pulmonary disease, idiopathic pulmonary fibrosis and healthy subjects. Respir Med. 2003;97:634-9.

19. Yoshioka A, Betsuyaku T, Nishimura M, Miyamoto K, Kondo T, Kawakami Y. Excessive neutrophil elastase in bronchoalveolar lavage fluid in subclinical emphysema. Am J Respir Crit Care Med. 1995;152:2127-32.

20. Kim D, Haynes CL. On-chip evaluation of neutrophil activation and neutrophil-endothelial cell interaction during neutrophil chemotaxis. Anal Chem. 2013;85:10787-96.

21. Friedrichs B, Neumann U, Schuller J, Peck MJ. Cigarette-smoke-induced priming of neutrophils from smokers and non-smokers for increased oxidative burst response is mediated by TNF-alpha. Toxicol In Vitro. 2014;28:1249-58.

22. Noguera A, Batle S, Miralles C, Iglesias J, Busquets X, MacNee W, Agusti AG. Enhanced neutrophil response in chronic obstructive pulmonary disease. Thorax. 2001;56:432-7.

23. Noguera A, Busquets X, Sauleda J, Villaverde JM, MacNee W, Agusti AG. Expression of adhesion molecules and $G$ proteins in circulating neutrophils in chronic obstructive pulmonary disease. Am J Respir Crit Care Med. 1998;158:1664-8.

24. Di Stefano A, Caramori G, Gnemmi I, Contoli M, Bristot L, Capelli A, Ricciardolo FL, Magno F, D'Anna SE, Zanini A, et al. Association of increased CCL5 and CXCL7 chemokine expression with neutrophil activation in severe stable COPD. Thorax. 2009;64:968-75.

25. Blidberg K, Palmberg L, James A, Billing B, Henriksson E, Lantz AS, Larsson K, Dahlen B. Adhesion molecules in subjects with COPD and healthy nonsmokers: a cross sectional parallel group study. Respir Res. 2013;14:47.

26. Boschetto P, Quintavalle S, Zeni E, Leprotti S, Potena A, Ballerin L, Papi A Palladini G, Luisetti M, Annovazzi L, et al. Association between markers of emphysema and more severe chronic obstructive pulmonary disease. Thorax. 2006;61:1037-42.

27. Paone G, Conti V, Vestri A, Leone A, Puglisi G, Benassi F, Brunetti G, Schmid G, Cammarella I, Terzano C. Analysis of sputum markers in the evaluation of lung inflammation and functional impairment in symptomatic smokers and COPD patients. Dis Markers. 2011;31:91-100.

28. Volpi G, Facchinetti F, Moretto N, Civelli M, Patacchini R. Cigarette smoke and alpha, beta-unsaturated aldehydes elicit VEGF release through the p38 MAPK pathway in human airway smooth muscle cells and lung fibroblasts. $\mathrm{Br} J$ Pharmacol. 2011;163:649-61.

29. Mortaz E, Henricks PA, Kraneveld AD, Givi ME, Garssen J, Folkerts G. Cigarette smoke induces the release of CXCL-8 from human bronchial epithelial cells via TLRs and induction of the inflammasome. Biochim Biophys Acta. 2011;1812:1104-10.

30. Swain ML, Eisner A, et al. Ultraviolet absorption spectra of nicotine, nornicotine and some of their derivatives. J Am Chem Soc. 1949;71:1341-5.

31. Clayton PM, Vas CA, Bui TT, Drake AF, McAdam K. Spectroscopic studies on nicotine and nornicotine in the UV region. Chirality. 2013;25:288-93.

32. Singh D, Edwards $L$, Tal-Singer $R$, Rennard S. Sputum neutrophils as a biomarker in COPD: findings from the ECLIPSE study. Respir Res. 2010;11:77.

33. Koethe SM, Kuhnmuench JR, Becker CG. Neutrophil priming by cigarette smoke condensate and a tobacco anti-idiotypic antibody. Am J Pathol. 2000;157:1735-43.

34. Finkelstein El, Nardini M, van der Vliet A. Inhibition of neutrophil apoptosis by acrolein: a mechanism of tobacco-related lung disease? Am J Physiol Lung Cell Mol Physiol. 2001;281:L732-739.

35. Milara J, Lluch J, Almudever P, Freire J, Xiaozhong Q, Cortijo J. Roflumilast $\mathrm{N}$-oxide reverses corticosteroid resistance in neutrophils from patients with chronic obstructive pulmonary disease. J Allergy Clin Immunol. 2014;134:314-22.

36. Iho S, Tanaka Y, Takauji R, Kobayashi C, Muramatsu I, Iwasaki H, Nakamura K, Sasaki Y, Nakao K, Takahashi T. Nicotine induces human neutrophils to produce IL-8 through the generation of peroxynitrite and subsequent activation of NF-kappaB. J Leukoc Biol. 2003;74:942-51.

37. Hastings J, de Matos P, Dekker A, Ennis M, Harsha B, Kale N, Muthukrishnan V, Owen G, Turner S, Williams M, Steinbeck C. The ChEBI reference database and ontology for biologically relevant chemistry: enhancements for 2013. Nucleic Acids Res. 2013;41:D456-463.

38. Russell RE, Culpitt SV, DeMatos C, Donnelly L, Smith M, Wiggins J, Barnes PJ. Release and activity of matrix metalloproteinase- 9 and tissue inhibitor of metalloproteinase-1 by alveolar macrophages from patients with chronic obstructive pulmonary disease. Am J Respir Cell Mol Biol. 2002;26:602-9.

39. Stevens JF, Maier CS. Acrolein: sources, metabolism, and biomolecular interactions relevant to human health and disease. Mol Nutr Food Res. 2008;52:7-25.

40. Facchinetti F, Amadei F, Geppetti P, Tarantini F, Di Serio C, Dragotto A, Gigli PM, Catinella S, Civelli M, Patacchini R. Alpha, beta-unsaturated aldehydes in cigarette smoke release inflammatory mediators from human macrophages. Am J Respir Cell Mol Biol. 2007;37:617-23.

41. Moretto N, Bertolini S, ladicicco C, Marchini G, Kaur M, Volpi G, Patacchini R, Singh D, Facchinetti F. Cigarette smoke and its component acrolein augment IL-8/CXCL8 mRNA stability via p38 MAPK/MK2 signaling in human pulmonary cells. Am J Physiol Lung Cell Mol Physiol. 2012;303:L929-938.

42. Fernandez-Nieto M, Quirce S, Fraj J, del Pozo V, Seoane C, Sastre B, Lahoz C, Sastre J. Airway inflammation in occupational asthma caused by styrene. J Allergy Clin Immunol. 2006;117:948-50.

43. Roder-Stolinski C, Fischader G, Oostingh GJ, Feltens R, Kohse F, von Bergen M, Morbt N, Eder K, Duschl A, Lehmann I. Styrene induces an inflammatory response in human lung epithelial cells via oxidative stress and NF-kappaB activation. Toxicol Appl Pharmacol. 2008;231:241-7.

44. Fischader G, Roder-Stolinski C, Wichmann G, Nieber K, Lehmann I. Release of MCP-1 and IL-8 from lung epithelial cells exposed to volatile organic compounds. Toxicol In Vitro. 2008;22:359-66.

45. Song MK, Choi HS, Lee HS, Ryu JC. Transcriptome profile analysis of saturated aliphatic aldehydes reveals carbon number-specific molecules involved in pulmonary toxicity. Chem Res Toxicol. 2014;27:1362-70.

46. Ludwig A, Petersen F, Zahn S, Gotze O, Schroder JM, Flad HD, Brandt E. The CXC-chemokine neutrophil-activating peptide-2 induces two distinct optima of neutrophil chemotaxis by differential interaction with interleukin-8 receptors CXCR-1 and CXCR-2. Blood. 1997;90:4588-97.

47. Schmidt T, Zundorf J, Gruger T, Brandenburg K, Reiners AL, Zinserling J, Schnitzler N. CD66b overexpression and homotypic aggregation of human peripheral blood neutrophils after activation by a gram-positive stimulus. J Leukoc Biol. 2012;91:791-802.

48. Dianzani C, Collino M, Lombardi G, Garbarino G, Fantozzi R. Substance P increases neutrophil adhesion to human umbilical vein endothelial cells. $\mathrm{Br}$ J Pharmacol. 2003;139:1103-10.

49. Schroder AK, Uciechowski P, Fleischer D, Rink L. Crosslinking of CD66B on peripheral blood neutrophils mediates the release of interleukin- 8 from intracellular storage. Hum Immunol. 2006;67:676-82.

50. Kang MJ, Oh YM, Lee JC, Kim DG, Park MJ, Lee MG, Hyun IG, Han SK, Shim YS, Jung KS. Lung matrix metalloproteinase-9 correlates with cigarette smoking and obstruction of airflow. J Korean Med Sci. 2003;18:821-7.

51. Culpitt SV, Rogers DF, Traves SL, Barnes PJ, Donnelly LE. Sputum matrix metalloproteases: comparison between chronic obstructive pulmonary disease and asthma. Respir Med. 2005;99:703-10.

52. Renda T, Baraldo S, Pelaia G, Bazzan E, Turato G, Papi A, Maestrelli P, Maselli $R$, Vatrella A, Fabbri LM, et al. Increased activation of p38 MAPK in COPD. Eur Respir J. 2008;31:62-9.

53. Jiang D, Wenzel SE, Wu Q, Bowler RP, Schnell C, Chu HW. Human neutrophi elastase degrades SPLUNC1 and impairs airway epithelial defense against bacteria. PLoS One. 2013;8:e64689.

54. Sussan TE, Gajghate S, Thimmulappa RK, Ma J, Kim JH, Sudini K, Consolini N, Cormier SA, Lomnicki S, Hasan F, et al. Exposure to electronic cigarettes impairs pulmonary anti-bacterial and anti-viral defenses in a mouse model. PLoS One. 2015;10:e0116861.

55. Gaffey K, Reynolds S, Plumb J, Kaur M, Singh D. Increased phosphorylated p38 mitogen-activated protein kinase in COPD lungs. Eur Respir J. 2013;42:28-41. 